tories, Pasadena, California, operated jointly with the California Institute of Technology.

Research in the astronomical field during the year included the discovery of more information about quasars, and the discovery of a probable physical inter-galactic connexion between our galaxy and the Magellanic Clouds-scientists from the Instituto Argentino de Radioastronomía working at the radio telescope constructed by the institution at La Plata, Argentina, investigated what seems to be a bridge of neutral hydrogen between the Milky Way and the small Magellanic Cloud.

Workers at the Department of Terrestrial Magnetism have found that the Earth's crust is very thin around Washington, DC. They have constructed a map showing the thickness of the crust from North Carolina to central Pennsylvania. The map shows that the crust varies in thickness from 30 to 60 kilometres, with the thinnest area underlying Washington and adjacent Virginia, and the thickest areas beneath the Appalachian Mountains. The Biophysics Section of this same department is interested in the biochemistry of evolution. It has reported work on the search for repeated DNA sequences in different species representing all the main forms of life, from viruses to humans. The investigators found that fifty-five different DNAs from plants and animals showed DNA sequence repetition. In other words, in each case, a large fraction of the genetic material consists of many copies of short DNA sequences or packets of genetic information-only bacterial and viral DNA failed to show sequence repetition. It is believed that the repeated DNA sequences are slowly mutated or changed during the course of evolution. Thus the copies (members of families of repeated sequences) do not remain identical. The degree of divergence may be a measure of the age of the family of nucleotide sequences-this divergence may be very relevant to evolutionary history. From time to time certain segments of DNA are copied and new families of many identical members are produced.

Among other research reported is the discovery at the Department of Plant Pathology of the way to make some common plants double their growth by reducing the amount of oxygen in the air surrounding them.

\section{New Research Rocket}

THw proving trials of a new British research rocket have been completed and a series of test flights have started in which scientific experiments are carried. The rocket is the Petrel, a small solid fuel vehicle designed to provide high altitude research facilities, mainly for universities, at low cost. It was developed for the Science Research Council by the Ministry of Technology's Rocket Propulsion Establishment, the Atomic Weapons Research Establishment and Bristol Aerojet Ltd. Petrel can reach altitudes of up to $140 \mathrm{~km}$ and can carry a payload of 13 kilograms, which has to be reduced if telemetry facilities are required.

The first of the series of test flights was launched successfully on February 3 and a second firing took place on February 29, both from the South Uist range in the Hebrides. The experiments carried on these flights were designed by the Radio and Space Research Station at Slough to investigate high speed electrons

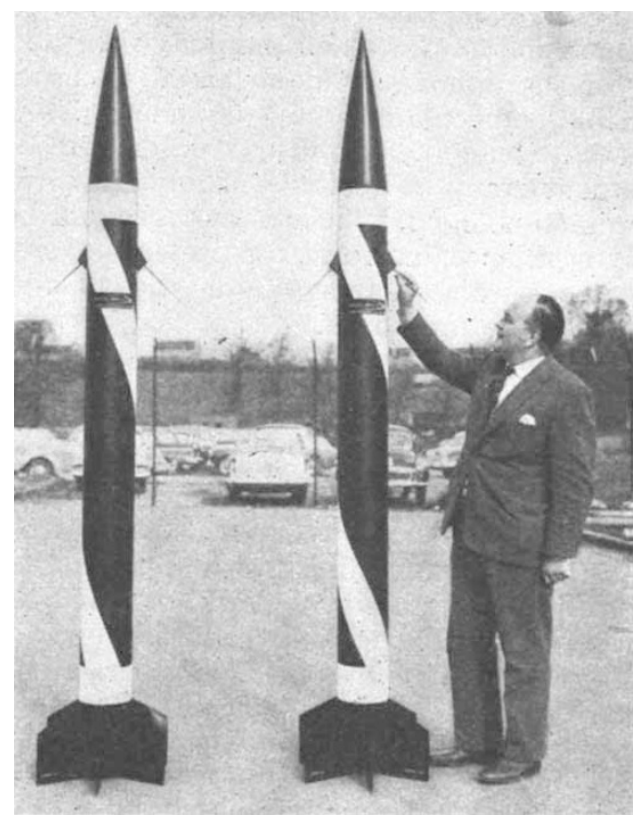

from space bombarding the atmosphere. The flights were tests in the sense that their purpose was primarily to evaluate the behaviour of the rocket rather than to gather scientific information.

Several more Petrel rockets are planned to be launched from South Uist this spring, carrying experiments for university research groups. These include payloads from Birmingham University to investigate ions and electrons in the atmosphere, from Southampton to study sporadic layers of ionization and from Sheffield to study fine structure effects in the ionosphere.

\section{Rules for Heart Transplants}

THE Board on Medicine of the US National Academy of Sciences has issued a set of guidelines for heart transplants. In a statement published on February 28 it says that because cardiac transplantation in man cannot yet be regarded as an accepted form of therapy, but only as a scientific exploration of the unknown, the operation should only be carried out in institutions where a special set of criteria can be met. The surgical team, the statement says, should have extensive laboratory experience of heart transplantation and a thorough understanding of the biological processes involved in rejection and its control-immunologists should be readily available to collaborate in the operation.

The statement goes on to say that the primary justification for transplanting hearts, with respect to donor and recipient, is that the knowledge so gained will be of benefit to other members of society. The ethical issues involved are part of the whole problem of the ethics of human experimentation, which are at present being studied intensely, as the statement says, by a number of well-qualified groups in the United States and other countries. Until some ethical guidelines are available the Board on Medicine can only advise every institution to assure itself that it has protected the interests of all parties involved to the fullest possible extent. The donor, the board says, should be unanimously 